\title{
Brain Circuits for the Internal Monitoring of Movements*
}

\author{
Marc A. Sommer ${ }^{1}$ and Robert H. Wurtz ${ }^{2}$ \\ ${ }^{1}$ Department of Neuroscience, the Center for the Neural Basis of Cognition, and the Center \\ for Neuroscience, University of Pittsburgh, Pittsburgh, Pennsylvania 15260; \\ email: masommer@pitt.edu \\ ${ }^{2}$ Laboratory of Sensorimotor Research, National Eye Institute, National Institutes \\ of Health, Bethesda, Maryland 20892; email: bob@lsr.nei.nih.gov
}

Annu. Rev. Neurosci. 2008. 31:317-38

First published online as a Review in Advance on April 2, 2008

The Annual Review of Neuroscience is online at neuro.annualreviews.org

This article's doi:

10.1146/annurev.neuro.31.060407.125627

Copyright (c) 2008 by Annual Reviews.

All rights reserved

0147-006X/08/0721-0317\$20.00

*The U.S. Government has the right to retain a nonexclusive, royalty-free license in and to any copyright covering this paper.

\section{Key Words}

vision, saccadic eye movements, corollary discharge, efference copy, perception
Abstract
Each movement we make activates our own sensory receptors, thus causing a problem for the brain: the spurious, movement-related sensa- tions must be discriminated from the sensory inputs that really matter, those representing our environment. Here we consider circuits for solv- ing this problem in the primate brain. Such circuits convey a copy of each motor command, known as a corollary discharge (CD), to brain regions that use sensory input. In the visual system, CD signals may help to produce a stable visual percept from the jumpy images resulting from our rapid eye movements. A candidate pathway for providing CD for vision ascends from the superior colliculus to the frontal cortex in the primate brain. This circuit conveys warning signals about impend- ing eye movements that are used for planning subsequent movements and analyzing the visual world. Identifying this circuit has provided a model for studying CD in other primate sensory systems and may lead to a better understanding of motor and mental disorders. 


\section{Contents}

INTRODUCTION .............. 318

IDENTIFYING CD

IN A COMPLEX BRAIN......... 319

A Model System for Studying CD:

Saccades and Vision ............ 319

A CD Path to Frontal Cortex ...... 319

Four Criteria for Identifying CD . . 319

CD AND SEQUENTIAL

SACCADES

Influence of CD on Behavior:

Double-Step Saccades.......... 321

Inactivation of the CD Pathway.... 322

Successive Saccades Depend on CD ................. 322

CD AND VISUAL PERCEPTION . . 324

Influence of CD on Vision:

Shifting Receptive Fields ....... 324

Spatial Match Between

CD and Shifting RFs......... 325

Temporal Match Between

CD and Shifting RFs......... 327

Necessity of CD Signal

for Shifting RFs............ 328

Shifting RFs and Visual Stability ... 330

CD BEYOND VISION............ 331

The Nature of the CD Signal...... 331

CD and Human Disease.......... 332

CONCLUSION .................. 334

\section{INTRODUCTION}

A major function of nervous systems is to process sensory input to detect changes in the environment. Yet animals have evolved not only to analyze but also to act, and actions have a multifold impact on sensory processing. A cat may notice a movement in a field and pounce toward a mouse. The cat's locomotion causes its legs to brush against grass, evoking touch sensations that must be ignored to avoid withdrawal reflexes. At the same time, the cat's head and eye movements disrupt its percept of auditory and visual space. It seems rather remarkable that a mouse is ever caught. Even at rest, if a cat perks up its ears to hear something better, the action momentarily disrupts the very sense it is intended to help. Nervous systems require some way of addressing these myriad, movementrelated disruptions. One major mechanism is to provide warning signals to sensory systems from within the movement-generating systems themselves.

The importance of motor-to-sensory feedback may be emphasized by considering what happens if there is no such link. Imagine a security guard watching images of a room from a video camera. If a suspicious person enters, the guard can quickly recenter the camera by moving a joystick. This mobility comes at the price of momentary disorientation, however, because the entire image leaps across the monitor. An animal's eyes are its security cameras, and the brain encounters problems similar to those faced by the security guard. Approximately twice per second we move our eyes to inspect a new part of the visual scene, but we have no overt awareness that our visual input is constantly lurching about. We perceive the world as stable. The fundamental difference between the security camera and the eye is internal feedback of movement information. The visual system is not surprised by eye movements because it receives a warning signal about each one. This warning signal is corollary discharge (CD).

The hypothesis that $\mathrm{CD}$ exists in the service of vision is a venerable one (Grüsser 1995) and was discussed most influentially by von Helmholtz in the nineteenth century (1925), who argued for the importance of an "effort of will." The first strong experimental evidence for the hypothesis was presented in the twentieth century by two groups working independently. Sperry (1950) coined the term corollary discharge, and his contemporaries von Holst \& Mittelstaedt (1950) referred to essentially the same principle as efference copy. We use the term CD to refer to the general concept of neuronal signals that provide internal information about movements.

In this review we concentrate on the demonstration of $\mathrm{CD}$ in the primate brain and its contribution to perception and motor planning. 
Our discussion focuses on the visual and oculomotor system because that is where the physiological basis of CD is best understood. We omit some phenomena that likely depend on CD because little circuit-level data exist, and we do not cover equally important aspects of CD revealed in studies of invertebrates and simpler vertebrate systems. We briefly consider the relevance of CD to other systems in the brain and to human disease.

\section{IDENTIFYING CD IN A COMPLEX BRAIN}

\section{A Model System for Studying CD: Saccades and Vision}

In our example of the security camera, we emphasized the disruption of vision as the camera shifted rapidly from one point of the scene to another. For the eyes, such rapid movements are called saccades. Saccades play the essential role in vision of moving the fine grain analyzer of the retina, the fovea, onto salient objects in the visual scene. The generation of saccades is among the more thoroughly understood motor processes of the primate brain. The saccadic network therefore provides an attractive model system for investigating the sources of CD and the pathways that distribute $\mathrm{CD}$ to sensory areas.

The visuosaccadic network extends from retinal registration of visual input to muscle contraction for movement output (Figure 1a). Visual input arrives at area V1 in cerebral cortex, and movement output descends from the superior colliculus (SC), through the midbrain and pontine reticular formation, to the oculomotor nuclei that innervate each of the six eye muscles. In between these primary input and output pathways lie cortical processing networks that range from $\mathrm{V} 1$ through subsequent visual processing areas (Figure 1 $\boldsymbol{a}$, yellow arrows), and which include the lateral intraparietal (LIP) area of parietal cortex and the frontal eye field (FEF) of frontal cortex. These parietal and frontal areas contribute to the generation of voluntary saccades (see reviews by Andersen
\& Buneo 2002, Colby \& Goldberg 1999, Schall 2002). LIP, FEF, and associated cortical areas in turn project to the intermediate layers of the SC to influence lower motor pathways (Figure 1 $\boldsymbol{a}$, red arrows). Ancillary circuits (including one from FEF through the basal ganglia to SC) and a second visual pathway (from SC superficial layers through pulvinar to visual cortex) are not shown.

\section{A CD Path to Frontal Cortex}

Given this background about the saccadic system, we can now ask what a saccadic CD circuit should look like. In principle, a CD pathway should run counter to the normal downward flow of sensorimotor processing. One such pathway (Figure $\mathbf{1} \boldsymbol{b}$, red arrows) ascends from the intermediate layers of the SC to the medial dorsal nucleus (MD) of the thalamus and then to FEF in frontal cortex. Other pathways may convey CD as well (gray arrows in the figure), but they have not yet been studied physiologically.

The pathway in Figure $\mathbf{1} \boldsymbol{b}$ was elucidated anatomically (as discussed below), but nothing was known about it functionally. The key question was, does it carry CD? Pathways for $\mathrm{CD}$ have been studied at the single neuron level in simpler nervous systems (Poulet \& Hedwig 2007). In the highly complex primate brain, it is likely more of a challenge to establish that a neuron carries CD as opposed to a movement command. By definition both the command and the corollary should look the same. We therefore developed a set of criteria (Wurtz \& Sommer 2004) that, if met, would satisfy us that we were studying a CD pathway.

\section{Four Criteria for Identifying CD}

First, the CD must originate from a brain region known to be involved in movement generation, but it must travel away from the muscles. The SC-MD-FEF pathway met this criterion. The pathway originates in the intermediate layers of the SC, an area clearly
Saccade: eye movement that rapidly redirects the fovea

SC: superior colliculus (of midbrain)

LIP: lateral intraparietal area (of parietal cortex)

FEF: frontal eye field (of frontal cortex)

MD: medial dorsal nucleus (of thalamus) 
a

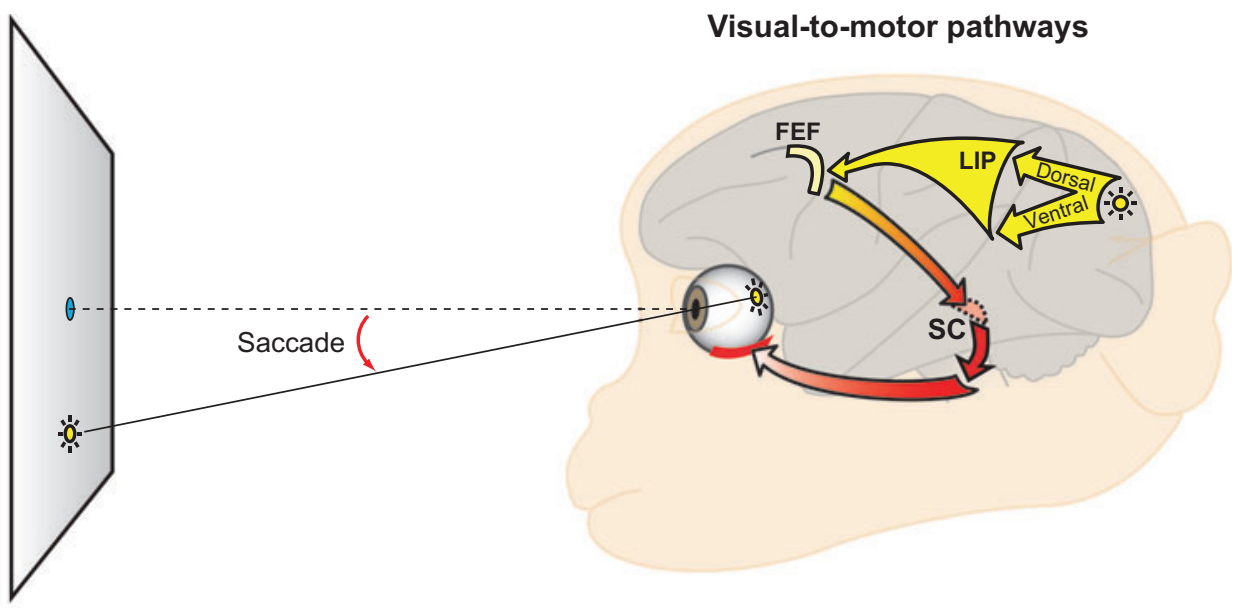

b

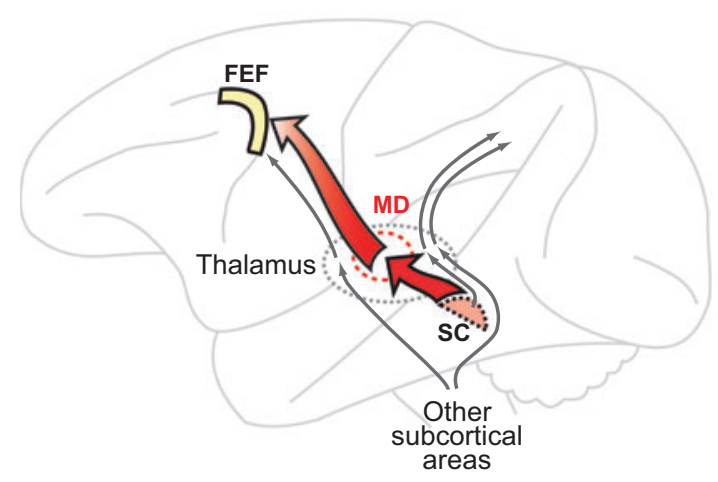

\section{CD signal}

Activity encodes when the saccade will start...

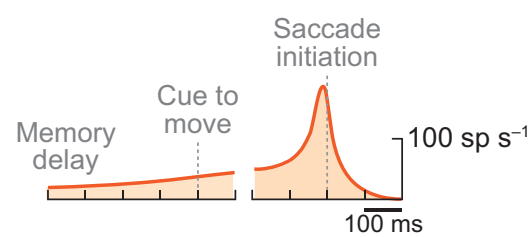

....and where the saccade will go.

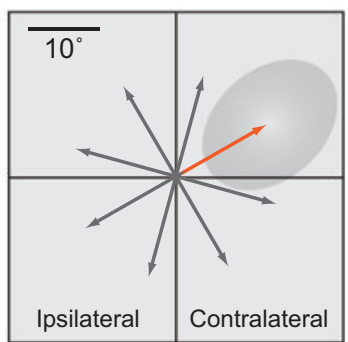

Figure 1

Visual and saccadic circuits in the monkey brain. (a) Outline of the major components of the circuit for generating visually guided saccades. Visual input arrives at area V1 of visual cortex ( far right) through a retinal-thalamic pathway (not shown). Visual signals (yellow) then are processed by dorsal and ventral cortical streams. A major intermediary region is LIP. Visual information from both streams converges in the FEF. Signals are sent downstream (red) to influence the SC and deeper motor areas that trigger a saccade. (b) Candidate pathways for conveying saccade-related CD to cerebral cortex. The SC-MD-FEF pathway has been studied in detail. Other routes may exist as well (gray arrows). (c) The CD signal in MD thalamic relay neurons that project to FEF. In a task used to characterize behavior-related activity of neurons, monkeys prepare saccades to the location of a remembered visual target. During the memory delay and cue to move periods, MD neurons display an increase in firing rate. This increase culminates in a burst of activity just before saccade initiation $(t o p)$. Spatially, the neurons fire only for specific ranges of contralateral saccades (bottom). 
involved in saccade generation. Neurons in the area discharge before saccades, electrical stimulation in the area produces saccades, and inactivation in the area disrupts saccades (Sparks \& Hartwich-Young 1989). Anatomical evidence for the pathway came from retrograde labeling and anterograde degeneration studies (Benevento \& Fallon 1975, Goldman-Rakic \& Porrino 1985) as well as trans-synaptic retrograde labeling using the herpes virus (Lynch et al. 1994). By combining the classic physiological techniques of antidromic and orthodromic stimulation, individual MD relay neurons were identified that received input from the SC and projected to the FEF (Sommer \& Wurtz 1998, 2004a). Taken together, these results confirmed that the pathway originates in a strongly saccade-related area but travels away from the eye muscles to innervate the frontal cortex.

Second, the CD signal should occur just prior to the movement and represent its spatial and temporal parameters. Approximately three quarters of the MD neurons identified as relay neurons increased their activity just before the saccade (Figure 1c, top) (Sommer \& Wurtz 2004a). Most MD neurons had peak activity for a limited range of saccadic amplitudes and directions, with strongest activity always occurring for directions into the contralateral visual field (Figure 1c, bottom). Thus the second criterion, a functional one, was met; neurons in this pathway are active before saccades and convey information about the parameters of the upcoming saccade.

Third, the neurons should not contribute to producing the movement. Silencing the pathway should not affect movements in simple tasks that do not require a CD. In accord with this criterion, inactivation of MD relay neurons did not alter saccadic accuracy and speed (Sommer \& Wurtz 2002, 2004b).

The fourth criterion was the most critical: It required that perturbing the putative $\mathrm{CD}$ pathway does disrupt performance on a task that depends on a CD. Perturbing the CD pathway did disrupt performance in such a task. This evidence was so central to illuminating the nature of the CD that we devote the next section to these experiments.

\section{AND SEQUENTIAL SACCADES}

\section{Influence of CD on Behavior: Double-Step Saccades}

In order to evaluate whether CD influences behavior, other factors affecting that behavior must be eliminated. First, the behavior under study must prohibit visual feedback about performance because primates use visual information whenever it is available. Elimination of visual feedback is achieved in the double-step task (Hallett \& Lightstone 1976), which has become standard in both the laboratory and the clinic. In this task, two targets are flashed sequentially. The subject's task is to make a saccade to the first target and then to the second. The first target may be flashed to the right, for example, and then the second target straight upward from there (Figure 2a, yellow dots). In correct performance, saccades would follow the targets as depicted by the solid arrows in the figure. No visual feedback is available during the task because the saccades are performed in total darkness; the fixation point and the two flashed targets are gone before the saccades begin. If the second saccade were made only on the basis of remembered visual information, it would go up and $45^{\circ}$ to the right (Figure $2 a$, dashed arrow) because that was the target's location when the eye was originally fixating. To make the correct second saccade, the memory of the target location must be adjusted to account for where the eye lands at the end of the first saccade. Internal information about the execution of the first saccade is needed. Because such information is not provided by visual feedback and is unlikely to come from proprioception (see Proprioception in sidebar), CD must be the source.

Quantifying the influence of CD on behavior requires an observable measure of that influence. CD itself is an internal signal and hidden from direct psychophysical assessment. In the double-step task, the observable event is the second saccade, and the parameter of interest is 


\section{PROPRIOCEPTION}

When visual feedback is absent or unreliable, extraretinal signals are required to monitor eye position. $\mathrm{CD}$ is one such signal, and proprioception of eye muscles is another (Wang et al. 2007). Although CD is of central origin and reaches cerebral cortex before a saccade, proprioception arises peripherally and reaches the cortex after a saccade. A series of experiments has shown that proprioception provides negligible information for everyday saccadic behavior. The common approach was to use microstimulation to displace a monkey's eye position while it prepared a voluntary saccade to a visual target. The question was, could a monkey internally monitor the displacement and compensate for it? The extent of compensation indicated the availability of extraretinal information. Stimulating the muscle-innervating motor neurons caused an eye displacement but no compensation, even though proprioception was available (Mays et al. 1987, Schiller \& Sandell 1983, Sparks \& Mays 1983). But stimulating higher in the circuitry, e.g., in the SC, caused a saccade that was immediately followed by full compensation, presumably because a concomitant CD signal was evoked. Compensation following SC stimulation persists even after the proprioceptive nerves are cut (Guthrie et al. 1983). Proprioception therefore seems insufficient as an extraretinal signal for making sequential saccades; instead, it may contribute to longer-term adjustments (Lewis et al. 2001, Steinbach 1987). its vector. A second saccade vector that lands at the second target location, fully compensating for the first saccade, implies perfect CD. Inaccuracies of the second saccade reveal inaccuracies of CD.

\section{Inactivation of the CD Pathway}

The hypothesis that the pathway to frontal cortex provides $\mathrm{CD}$ information about the first saccade was tested by reversibly inactivating the $\mathrm{MD}$ relay node (Figure $\mathbf{2} \boldsymbol{b}$ ) in the pathway. This left both the SC and FEF untouched while severing the link from SC to FEF (Sommer \& Wurtz 2002, 2004b). If inactivation totally eliminates $C D$, the monkey should make the first saccade correctly but should not have internal information that it did so. In the absence of information about this first saccade, the sec- ond saccade should be inaccurate; it should be directed toward the initial, retinotopic location of the second visual target. The average end points of second saccades were analyzed to see how they changed during inactivation.

Inactivation of MD did cause second saccade end points to shift as if CD of first saccades was impaired, as long as those first saccades were directed into the contralateral hemifield (Figure 2c). The impairment was significant but smaller than expected from total CD elimination. This partial deficit suggests that we did not silence the path completely or that other CD pathways exist. Both hypotheses are reasonable but have yet to be tested.

What makes the results more compelling is that the deficit was limited to trials in which the first saccades were made into the contralateral visual field (Figure $2 c$, right). This direction was the same preferred direction of the MD relay neurons. Double-step configurations in which first saccades went into the ipsilateral visual field were randomly interleaved, and these trials were unaffected (Figure $2 c$, left). In sum, there was a modest deficit in the average accuracy of second saccades during inactivation, but the deficit matched the direction of saccades represented in the affected MD nucleus.

Similar double-step deficits have been found in patients subsequent to thalamic injury (Bellebaum et al. 2005, Gaymard et al. 1994), parietal injury (Duhamel et al. 1992b, Heide et al. 1995), and parietal transcranial magnetic stimulation (Morris et al. 2007). Such deficits have not been reported for human FEF lesions, but the major confound that FEF damage causes impairment to saccade generation complicates analysis of CD deficits (discussed in Sommer \& Wurtz 2004b).

\section{Successive Saccades Depend on CD}

A finer-grained analysis of the data, focusing on the precision of individual saccades, revealed a more striking deficit (Sommer \& Wurtz 2004b). During normal behavior, monkeys made first saccades that landed at slightly different places 


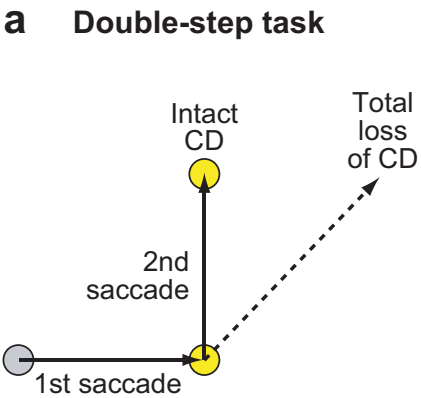

d

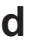

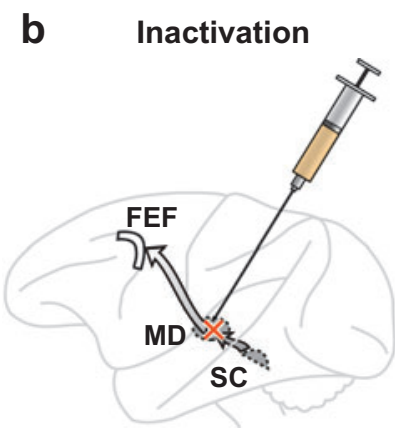

C

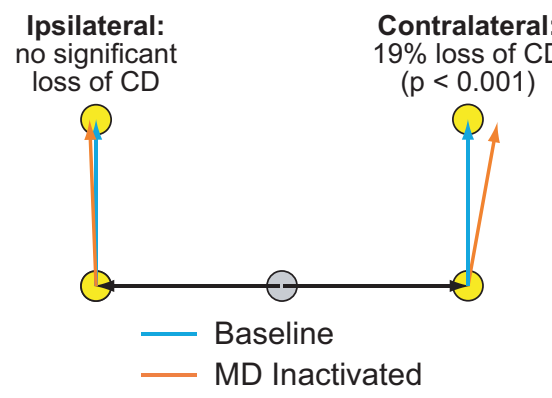

Accuracy deficit

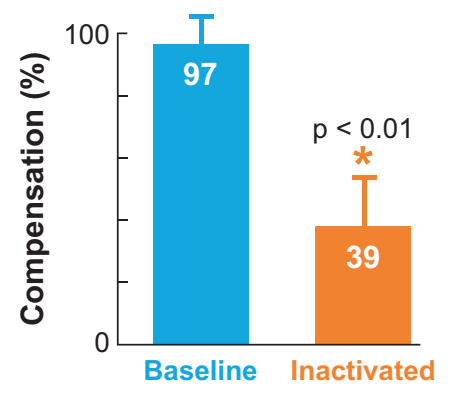

Figure 2

Double-step task used to evaluate the influence of CD. (a) The pattern of double-step saccades (arrows) that would be expected with intact CD vs. total loss of CD. (b) Inactivation of the putative CD pathway: the GABA agonist muscimol was injected at the site of MD relay neurons. $(c)$ Average accuracy deficit, measured as the horizontal shift in second saccade endpoints. Trials with contralateral first saccades (right) showed a clear deficit, but ipsilateral first saccades did not produce a deficit $(l e f t)$. $(d)$ Precision deficit in compensating for trial-by-trial variability in first saccades. Compensation is illustrated for second saccade vectors in an example session. The vectors are spread out in the vertical dimension for clarity, but their relative horizontal positions are maintained (as indicated by axis at bottom). Deficit across all sessions $(n=22)$ is summarized with the bar graph (inset). Bars show means and standard errors; on average there was a $58 \%$ deficit ([97\%-39\%]/97\%).

from trial to trial: sometimes short of where the first flashed target had been, sometimes beyond it. It therefore seemed likely that if a CD occurs with each saccade as predicted from the neuronal activity found in $\mathrm{MD}$, a CD-driven compensation should also occur that is unique to each trial. As predicted, the individual second saccade vectors (Figure $\mathbf{2} \boldsymbol{d}$, blue lines) rotated forward or backward depending on where they started (i.e., where the first saccades landed). 
RF: receptive field

FF: future field
Hence in its normal state, the brain precisely monitors even small fluctuations in saccade amplitude and adjusts subsequent saccades accordingly.

Now if the CD were altered by inactivation, the compensation should be altered. This was, in fact, the case: the second saccades during inactivation (Figure $2 \boldsymbol{d}$, orange lines) failed to rotate as a function of where the first saccades ended. Compensation was reduced by more than half(Figure $2 \boldsymbol{d}$, right). Monkeys could still compensate slightly after MD inactivation, so the deficit was partial, as it was for the average accuracy deficit. And once again, the effect occurred only for saccades into the contralateral visual field.

These inactivation experiments suggest that activity in the SC-MD-FEF pathway not only correlates with what is expected of a CD signal, but actually functions as a CD signal. With this demonstration, the signals in the pathway from SC to FEF via MD satisfy the four criteria we regard as essential for identifying CD. The signals originate from a known saccaderelated region, they encode the timing and spatial parameters of upcoming saccades, their removal does not affect the generation of saccades for which $\mathrm{CD}$ is not required, and their removal does disrupt saccades for which $\mathrm{CD}$ is required.

\section{AND VISUAL PERCEPTION}

\section{Influence of CD on Vision: Shifting Receptive Fields}

Having reviewed evidence that the information conveyed upward through the SC-MDFEF pathway is a CD signal, we now return to the fundamental hypothesis: that the world appears stable across eye movements because CD informs the visual system about each imminent saccade. Whether cortical visual neurons truly use a CD signal was unknown until landmark experiments on parietal cortical area LIP by Duhamel et al. (1992a) revealed changes in neuronal activity that must result from CD input.
Neurons in the visual system have a restricted view of the world known as a receptive field (RF). The neurons respond only if light falls on a restricted zone of the retinathe RF-and conventional thought was that the zone's location changes little, if at all, when the eyes move. Duhamel et al. (1992a) found, however, that some neurons in LIP behave differently. A new zone of retinal sensitivity emerges at a location dependent on the saccade that is about to occur. Such a shifting RF is possible only if the neuron receives information about the imminent saccade.

The fundamental nature of a shifting RF is depicted in Figure $\mathbf{3} \boldsymbol{a}$. Suppose that during fixation (left panel), a neuron has an RF (blue ellipse) aligned directly below the fovea (orange dot). The neuron would respond to an apple in its RF but not to a distant pepper. If the monkey plans to make a saccade to the point just above the pepper, however (middle panel), a neuron with a shifting RF shows a crucial change. It suddenly becomes sensitive to the pepper (magenta ellipse) because the pepper lies at the location where the RF will reside after the saccadereferred to as the future field (FF). Then the eyes move (right panel), and the presaccadic FF becomes the RF again. Such shifting RFs (also called remapped RFs) have been studied in many regions including LIP (Batista et al. 1999, Colby et al. 1996, Duhamel et al. 1992a, Heiser \& Colby 2006, Kusunoki \& Goldberg 2003), FEF (Sommer \& Wurtz 2006; Umeno \& Goldberg 1997, 2001), extrastriate visual areas (Nakamura \& Colby 2002, Tolias et al. 2001), and the SC (Walker et al. 1994). They are likely present in the human brain as indicated by fMRI studies (Medendorp et al. 2003; Merriam et al. 2003, 2007), and their relation to corpus callosum function has been examined (Berman et al. 2005, 2007; Heiser et al. 2005).

Shifting RFs are not studied in the laboratory with apples and peppers, of course, but with well-controlled flashes of light (probes). The activity of a neuron with a shifting RF demonstrates its presaccadic change in visual sensitivity across space (Figure $3 \boldsymbol{b}$, an FEF neuron). During initial fixation, the neuron responded 
a

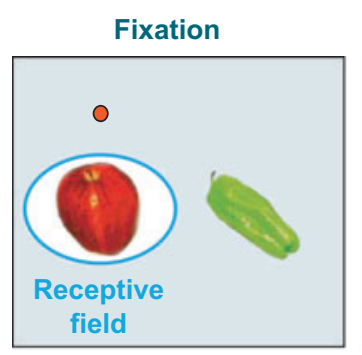

b

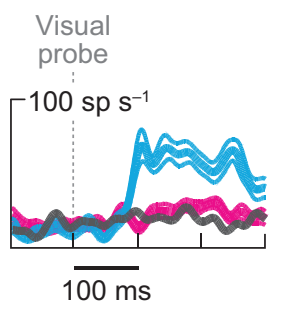

Shifting RFs: concept
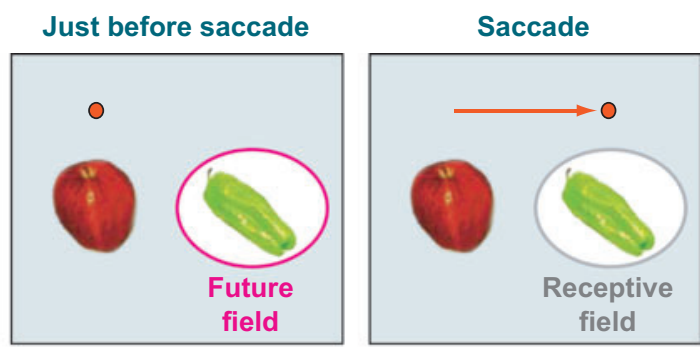

\section{Neuron}

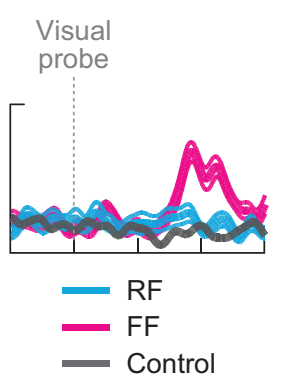

\section{Saccade initiation}

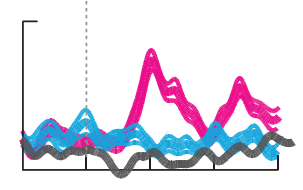

Figure 3

Shifting visual receptive fields. (a) Concept shown schematically. (b) Example of an FEF neuron that shows the shift. A visual probe was flashed in the RF in some trials (blue) or in the FF in other trials (magenta). No probe appeared in control trials (black). Curves show mean and SE of average neuronal activity aligned to probe onset (left and center panels) or to saccade initiation (right panel).

only to probes flashed at the RF (left) and just before the saccade only to probes flashed at the FF (middle). Because the visual activity associated with the FF stimulation appeared around the time of the saccade (right), a fair question is whether the activity was related to making the saccade. This possible artifact was tested with interleaved trials in which no probe appeared, but the same saccade was made (black traces). Such control trials elicited no activity. The activity required a visual stimulus in the FF.

Our hypothesis was that, in FEF neurons, shifting RFs are caused by CD input from the MD-mediated pathway. This hypothesis led to three predictions. First, the spatial characteristics of the shifting RF should reflect those of the CD. Second, the temporal dynamics of the shift should match those of the CD. If both of these first two predictions were confirmed, the CD would be established as appropriate for driving the shift. The third prediction was that the shift should be reduced if the CD were interrupted. Such a result would show that the CD is necessary for the shift.

\section{Spatial Match Between CD and Shifting RFs}

The first prediction about shifting RFs proposed that RFs should have spatial properties similar to the CD itself. The CD in the pathway to FEF originates in the SC (Figure 4a), a structure with exquisite topographic organization of saccade vectors. If SC activity provides the $\mathrm{CD}$ that causes the FEF shift, the shift should move as if prompted by a vector input. It should jump to its new location (Figure $4 b$ ) rather than spreading continuously from RF to FF. The prediction was testable by flashing probes not only at the RF and FF, but 
a

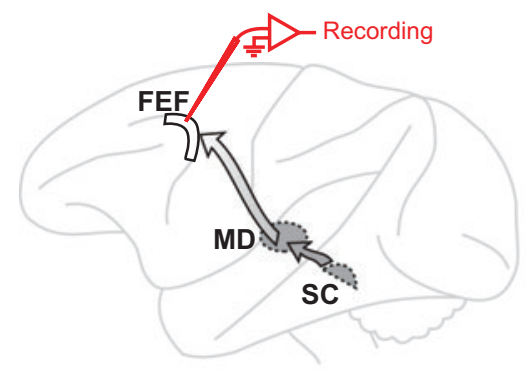

C

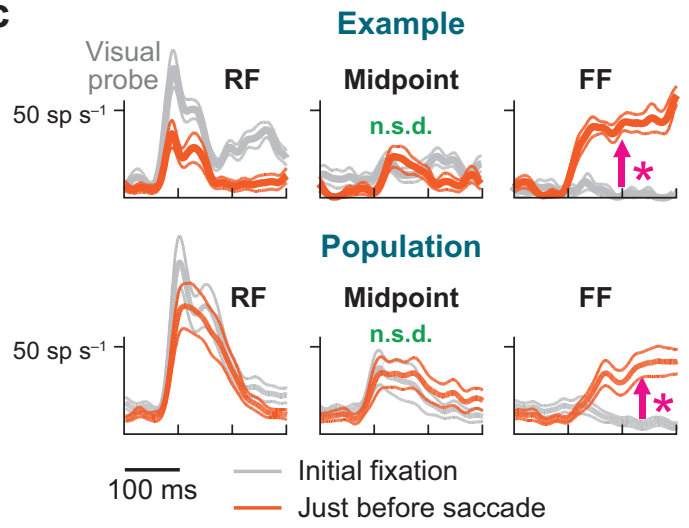

b

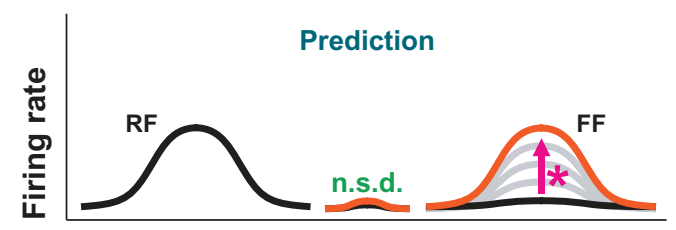

d
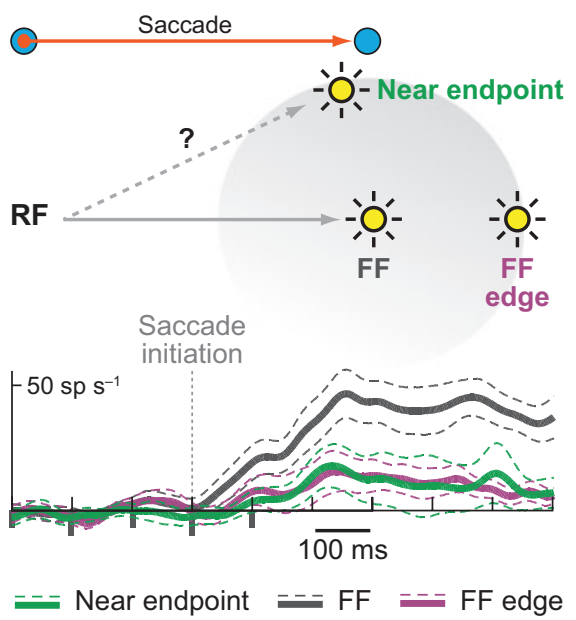

Figure 4

Shifting RFs: spatial. (a) Neurons with shifting RFs were recorded in the FEF. (b) It was predicted that shifting RFs would jump, as expected from a vector CD input. There should be a significant change in activity (asterisk) at the FF but no significant difference (n.s.d.) at the midpoint. (c) (Top) Example FEF neuron shows a jump, not a spread of activity. Just before a saccade, there was a decrease in activity at the RF (left), no change in activity at the midpoint (middle), and a large increase in activity at the FF (right). (Bottom) The same result was found in the average of the FEF population ( $n=13$ neurons). (d) Experiment to see if shifting RFs travel toward the saccadic endpoint ("?" trajectory). Shifting RF activity is shown for probes flashed near the endpoint (green), at the edge of the FF as defined by a shift parallel to the saccade (purple), and at the middle of the FF so defined (black). The data indicate that shift travels parallel to the saccade, not toward the saccadic endpoint (average data from $n=12$ neurons).

also at the midpoint between them. If the RFs jump, the midpoint would exhibit little or no activity; if they spread, the midpoint would become highly active.

Data from an example neuron (Figure $4 c$, top) revealed that while activity increased dramatically at the FF just before a saccade, no change occurred at the midpoint. The result from this example neuron was reproduced in the overall population (Figure $4 c$, bottom). The data supported a jump, not a spread, of activ- ity, compatible with a vector CD input. The small amount of activity at the midpoint, which did not change significantly before the saccade, was caused by the probe sometimes falling on the edges of the RF and FF (Sommer \& Wurtz 2006).

In this analysis the implicit assumption has been that the shifting RFs move parallel to the saccade as implied by the horizontal shift with a horizontal saccade in Figure $\mathbf{3} \boldsymbol{a}$. Shifting RFs in visual area $\mathrm{V} 4$, however, move toward the 
saccadic end point (Tolias et al. 2001). The direction of the shift is crucial because the FF location provides clues about the perceptual function of the shift, as discussed below. To examine the issue in FEF neurons, probes were introduced near the saccadic end point (Figure $4 d$, top). The shift at that location, however, was minimal (Figure $4 d$, bottom). It was no different from activity at the edge of the FF as defined by a parallel shift and much less than activity at the center of the FF so defined. The data supported our hypothesis that shifting RFs in the FEF move parallel to the saccade.

\section{Temporal Match Between $\mathrm{CD}$ and Shifting RFs}

The next prediction was that the timing of shifting RFs should match the timing of the $\mathrm{CD}$ signal in the SC-MD-FEF pathway. The recording study (Sommer \& Wurtz 2004a) demonstrated that the pathway conveys a gradual increase in activity and then a burst of activity aligned with saccade onset (see Figure 1c). If $\mathrm{CD}$ from the pathway causes the shift, the shift should be time-locked with the saccade.

We analyzed whether shifting RF activity was better related to stimulus onset or to saccade initiation. Shifting RF activity requires visual stimulation in the FF, and Figure $5 \boldsymbol{a}$ shows for one neuron the temporal profile of visual probe presentation in the $\mathrm{FF}$ and the neuronal activity that it evoked. The probe appeared at time 0 and disappeared after $50 \mathrm{~ms}$. The neuron showed no activity at its normal visual latency of $80 \mathrm{~ms}$. Rather, activity appeared much later, and with appreciable variability. In individual trials, the start of the activity seemed closely linked to the start of each saccade. The correlation between activity onset and saccade onset was highly significant for this neuron and for the population (Sommer $\&$ Wurtz 2006). Alignment of the average visual activity improved when the same data were plotted relative to saccade initiation (Figure $5 \boldsymbol{b}$, higher peak and narrower SEs). Thus a shifting $\mathrm{RF}$ is visual activity synchronized to a motor act.

\section{Shifting RFs: timing}

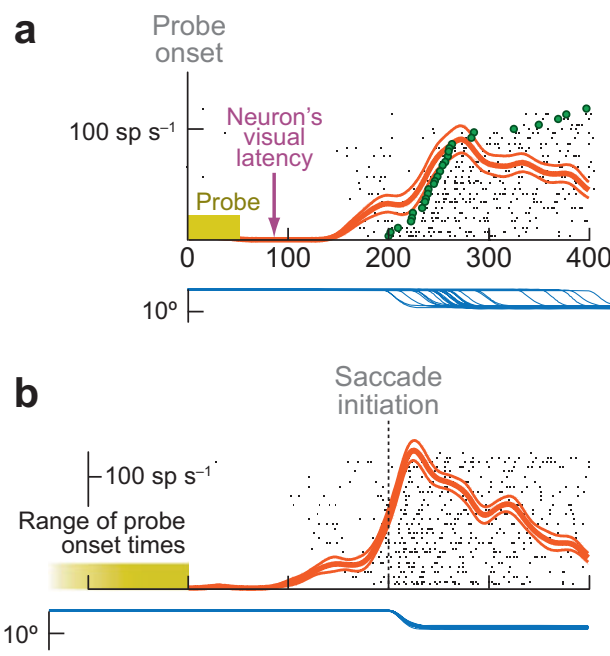

Figure 5

Shifting RFs: timing. (a) Shifting RF activity aligned to probe onset for an example neuron. Each raster shows action potentials from a single trial, and the bold orange trace is average firing rate (surrounded by thin traces showing standard errors). Start of the shift is much later than the neuron's normal visual latency (purple arrow). Also shown are times of saccade onset in each trial (green dots) corresponding to the eye position data (traces at bottom). (b) Shifting RF activity aligned to saccade initiation. For this neuron, shift onset time and saccadic onset time were correlated with Pearson $\mathrm{R}=0.97$; for the population, $\mathrm{R}=0.50$ (both $\mathrm{p}<0.01$ ).

Although saccade-gated visual activity may seem bizarre, it makes sense; if the shifting RF is concerned with maintaining perceptual visual stability, it would be important for it to occur only if the generation of the saccade were inevitable. Saccades can be cancelled up to $100 \mathrm{~ms}$ before their initiation (Hanes \& Schall 1995). The only way to ensure that shifts are linked to saccades, therefore, is to delay their onset to begin only after the "point of no return" for moving, i.e. $100 \mathrm{~ms}$ or less before saccade initiation.

Shifts were time-locked to saccades, but their exact onset times varied from neuron to neuron as indicated by four examples (Figure 6a). The shift started before the saccade began for some neurons and after the 


\section{Shifting RFs: timing of CD influence}
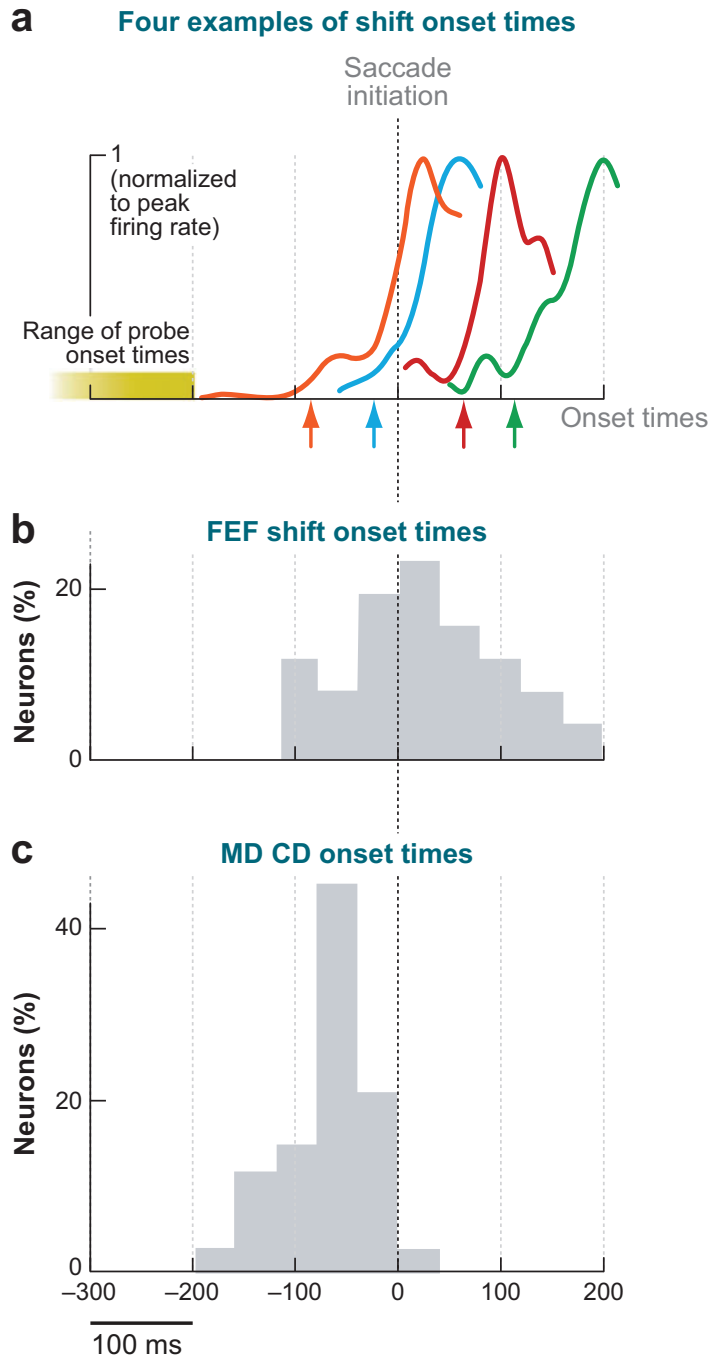

Figure 6

Shifting RFs: timing of CD influence. (a) Shift onset times for four example FEF neurons. Some neurons started their shift before saccade initiation (orange and blue traces), while others started their shift after saccade initiation (red and green traces). The orange trace is from the neuron in Figure $\mathbf{5} \boldsymbol{a}$, and the red trace is from the neuron in Figure $\mathbf{3} b$. The blue and green traces show data from two other neurons. Shift magnitudes are normalized to each other to facilitate comparison of timing. Note that the probe onset time always preceded saccade onset. (b) Distribution of shift onset times for the sample of FEF neurons $(n=26)$. (c) Distribution of CD onset times (the start of the saccade-related bursts) in the population of MD relay neurons that project to FEF $(n=33)$. saccade began for others. Yet for all the neurons, probes were flashed at identical times (yellow range to left) and always appeared and disappeared in the neuron's FF. Even though some shifts began after saccade initiation, they were caused by a visual event happening before saccade initiation and outside the classic RF.

Overall, the shift onset times for the neuronal population were distributed approximately normally, over a range of $100 \mathrm{~ms}$ before to $200 \mathrm{~ms}$ after saccade initiation (Figure $6 \boldsymbol{b}$ ). The average shift onset time was $24 \mathrm{~ms}$ after the saccade started, which is in midflight of a typical saccade. How does this distribution of shift onset times compare with the CD signal that we posit is causing the shift? Figure $6 \boldsymbol{c}$ shows the range of onset times for the $\mathrm{CD}$ signal that $\mathrm{MD}$ relay neurons provide to the FEF. Just as shift onset times in the FEF were measured by a sudden increase in visual activity of shifting neurons, CD onset times were measured by a sudden increase in MD relay neuron saccade-related activity. The average CD onset time was $72 \mathrm{~ms}$ before the saccade, demonstrating that the CD signal could be a causal trigger for the shift. It takes $\sim 100 \mathrm{~ms}$ $(72+24=96 \mathrm{~ms})$ for CDs arriving at the FEF to trigger a shifting RF. This delay implies that shifts are not constructed by single, MDrecipient FEF neurons, but rather that they develop through a multisynaptic network.

\section{Necessity of CD Signal for Shifting RFs}

The final prediction was that interrupting the CD signal should reduce the shift. Up to this point the comparison of CD to shift was one of spatial and temporal correlation; perturbing the $\mathrm{CD}$ allows us to move from correlation to causation. The CD signal was inactivated at the MD relay neuron level during continuous recording of an FEF neuron with a shifting RF (Figure 7a) (Sommer \& Wurtz 2006). If the prediction were correct, the FEF neuron would retain its classic visual response (in the RF) but lose its ability to shift the response (into the FF). 
b

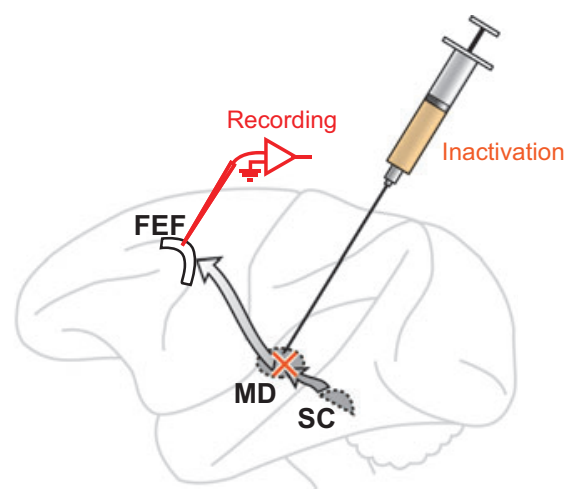

\section{Example}

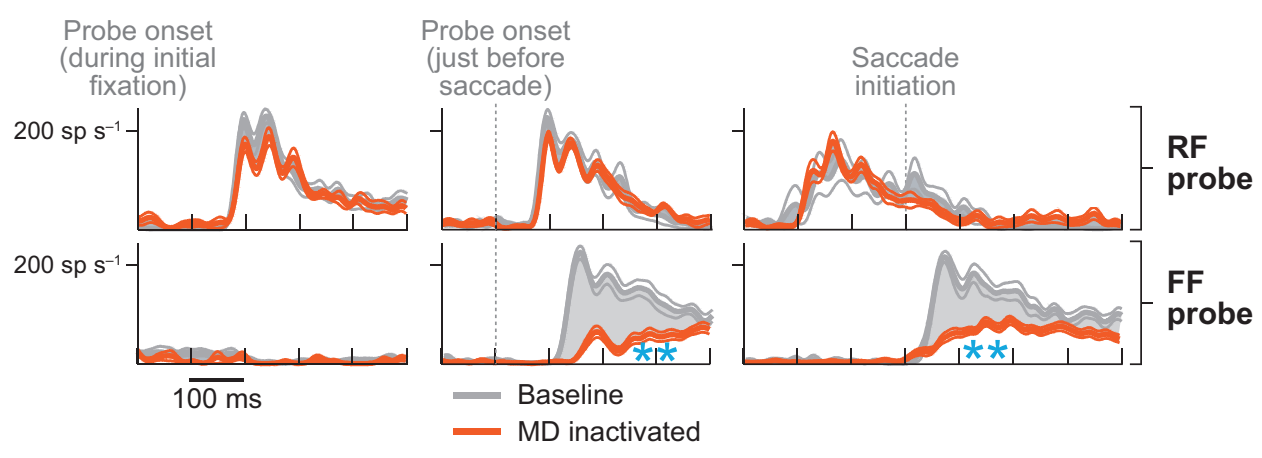

C
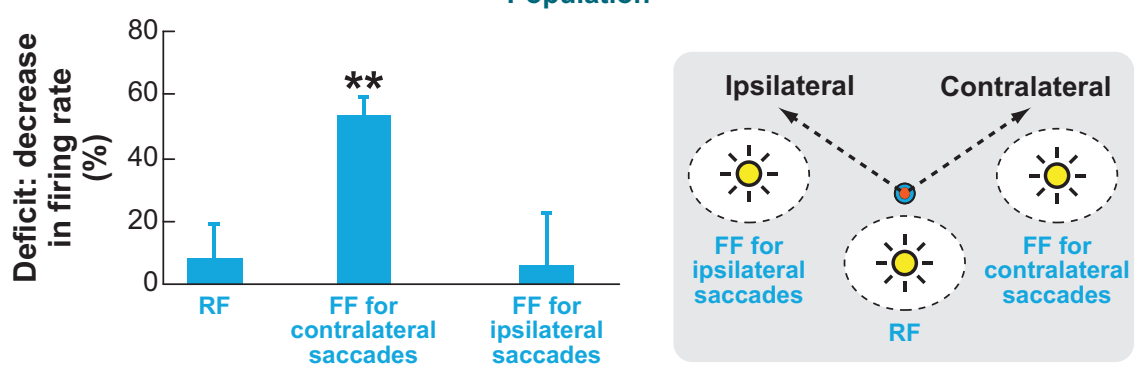

Figure 7

Shifting RFs: necessity of CD pathway. (a). Inactivation of the CD pathway while an FEF neuron with a shifting RF is recorded. (b) Example of a shifting RF that was impaired by MD inactivation. Activity before inactivation is depicted with grey traces. The neuron had a visual response in the RF during fixation (left panels), a shift to the FF just before the saccade (lower middle panel), and tight alignment of this shift with saccadic onset (right panel). The neuron maintained its activity in the RF even as it gained sensitivity at the FF, as many neurons did (upper middle panel). During inactivation (orange traces), the FF response was drastically reduced (bottom row). The classic RF response, however, was unchanged (top row). (c, left) The deficit in the population of neurons. Reductions in activity were seen only in the FF, not the RF, and only for contralateral, not ipsilateral, saccades. $(c$, right $)$ Task configurations for testing shifting RFs that accompanied contralateral saccades vs. ipsilateral saccades. ${ }^{* *}$, significant changes at $\mathrm{p}<0.0001$ level. 
Data from an example FEF neuron are shown in Figure $7 \boldsymbol{b}$. Before inactivation, the neuron had a strong visual response in the RF and an intense shift of visual sensitivity into the FF that was synchronized with the saccade. During inactivation, the FF response was drastically reduced even though the classic visual response in the RF was unchanged. In sum, when MD was inactivated, the FEF neuron retained its visual sensitivity and the monkey retained its ability to make a saccade, but the communication between saccade generation and visual processing was gone; the neuron lost advanced information about the saccade (i.e., CD), severely impairing its ability to shift visual sensitivity to the FF location.

The deficits found in the example neuron were replicated individually in most neurons tested (7 out of 8 ) and were highly significant in the pooled data (Figure $7 \boldsymbol{c}$, left). Overall, the shift was reduced by an average of $\sim 50 \%$ when it accompanied contralateral saccades, those represented by the SC-MD-FEF pathway. Shifts that accompanied ipsilateral saccades were unimpaired.

In summary, our predictions were supported: Shifting RFs had spatial and temporal dynamics consistent with the CD signal, and the shifts depended on the CD signal. This provided systematic evidence for a functional link between the CD arising from the midbrain and visual processing in the FEF. This result produces an intriguing further implication when considering the FEF's position in the broader network of cortical visual areas. The FEF may play a role in relaying CD information, or shifting RFs themselves, to other cortical areas such as LIP where shifting RFs are found. Functional, reciprocal connections do link FEF and parietal cortex (Chafee \& Goldman-Rakic 2000).

\section{Shifting RFs and Visual Stability}

The previous section and Figures 3-7 have described shifting RFs. Because of their unique characteristics, shifting RFs likely play a role in the perception of visual stability across sac- cades (Duhamel et al. 1992a). To see why this hypothesis is plausible, we must discuss in more detail how CD signals influence shifting RFs and how shifting RFs, in turn, could influence visual analysis.

The CD signals produce a spatial effect in the retinotopic coordinate system used for visual processing. This effect is a new receptivity to visual stimulation at the FF. The FF's location is determined by the vector of the impending saccade conveyed by the CD. How the motorto-sensory transformation between the saccadic system and the visual map could be made has been modeled by Quaia et al. (1998). For a neuron with a shifting $\mathrm{RF}$, the $\mathrm{CD}$ signal enables visual responsiveness at a specific location on the visual map, and the information conveyed by the neuron is about visual events in visual coordinates.

In temporal terms, saccadic CD has a gating effect on visual activity. A normal visual response to a stimulus in the RF has a brisk onset and fixed latency relative to stimulus onset. But activity evoked by a visual stimulus in the FF has no such brisk onset; as was shown in Figure 5, it is tied not to stimulus onset but to saccade onset. The visual activity may be held in check for as long as $1000 \mathrm{~ms}$ until the saccade begins (Umeno \& Goldberg 2001). Only around the time of the saccade is the visual activity released in a surge of action potentials. As noted above, saccadic gating of the FF visual activity is a strange but seemingly critical feature; it guarantees that a shifting RF occurs only if a saccade occurs.

The above description of shifting RFs confirms that the phenomenon has two phases: an early phase of visual sensitivity at the FF that can begin long before the saccade, and a later phase of unleashed visual activity that begins with the saccade. These two phases fit well with the nature of the CD signal (see Figure 1 $c$, top), which has a low-frequency, prelude phase that could induce visual sensitivity at the FF and a high-frequency, burst phase that could trigger the saccade-aligned visual activity.

Considering these shifting RF properties, we can evaluate the hypothesis that they 
contribute to the perception of visual stability (Colby \& Goldberg 1999, Sommer \& Wurtz 2006). We think the hypothesis is compelling because of a specific, key feature of shifting RFs: The presaccadic FF location and the postsaccadic RF location sample the same absolute location in visual space. For an illustration of this overlap, see Figure $\mathbf{3} \boldsymbol{a}$ and note that the FF location (middle box) is identical to the postsaccadic RF location (right box). Information is available, therefore, about a single region of the visual world before and after the saccade. If the presaccadic image equals the postsaccadic image, objects must have remained stable across the saccade. If there is a presaccadic-postsaccadic difference, objects must have moved while the eyes moved. Thus, shifting RFs could allow single neurons to assess the stability of the world across saccades.

Confirmation or refutation of this hypothesis calls for two lines of research. One would address in a conceptual manner how transsaccadic information provided by shifting RFs could lead to a percept of visual stability. Which exact computational principles are involved? Must an overt representation of stable space emerge from the comparison of presaccadic FF with postsaccadic RF, or can it remain implicit at a network level? Answering these questions will almost certainly depend on developing more sophisticated models of how neuronal activity leads to perceptual experiences.

The other line of research would empirically test the link between shifting RFs and perceptual stability. On this issue, the experiments on the SC-MD-FEF pathway provide a new opportunity. Because we have established that at the neuronal level we can disrupt the shifting RFs in FEF by interrupting the ascending pathway, we can now test whether interrupting this pathway also disrupts visual stability when saccades occur. Investigators would train a monkey to report when an object moves in front of it as opposed to when an object seems to move as a result of its own saccades. We predict that during inactivation of the CD pathway, the monkey will erroneously report that an object moves during a saccade when, in fact, it is still.
Such a demonstration would provide the final link in the mechanistic chain from identified CD signal, to the shifting RF phenomenon, to the percept of visual stability across saccades.

\section{BEYOND VISION}

\section{The Nature of the CD Signal}

Until recent years, the mechanistic nature of $\mathrm{CD}$ in the primate has largely been a matter of speculation. Now, with insight into one example of the neuronal basis of $\mathrm{CD}$, we can compare speculation with observation. Three salient concepts about how CD exerts its influences have been suppression, cancellation, and forward models.

The first CD concept is also the simplest: The CD simply suppresses visual information. Evidence for such suppression with saccades has been found in single neurons of the superficial visual layers in SC (Robinson \& Wurtz 1976). The suppression resulted from a CD rather than from proprioceptive feedback because preventing the proprioception did not eliminate the suppression (Richmond \& Wurtz 1980). This was the first demonstration of the CD's effect in the primate brain. The recent demonstration of an inhibitory input from the saccade-related intermediate layers of the SC to the visual superficial layers (Lee et al. 2007) suggests a mechanism for the effect. Clear suppression, which may also result from CD, has been demonstrated in the primate pulvinar (Robinson \& Petersen 1985) and extrastriate area MT (Thiele et al. 2002); the lateral geniculate nucleus shows only modest suppression (Ramcharan et al. 2001, Reppas et al. 2002, Royal et al. 2006).

The second concept, cancellation, was put forward most forcefully by von Holst \& Mittelstaedt (1950): "[T]he efference leaves an 'image' of itself somewhere in the CNS to which the reafference (i.e., sensory signs of the resulting movement) compares as the negative of a photograph compares to its print" (von Holtz quoted in MacKay 1972). Note that reafference is specifically due to self-movement,
Reafference: sensory inflow evoked by one's own movements 


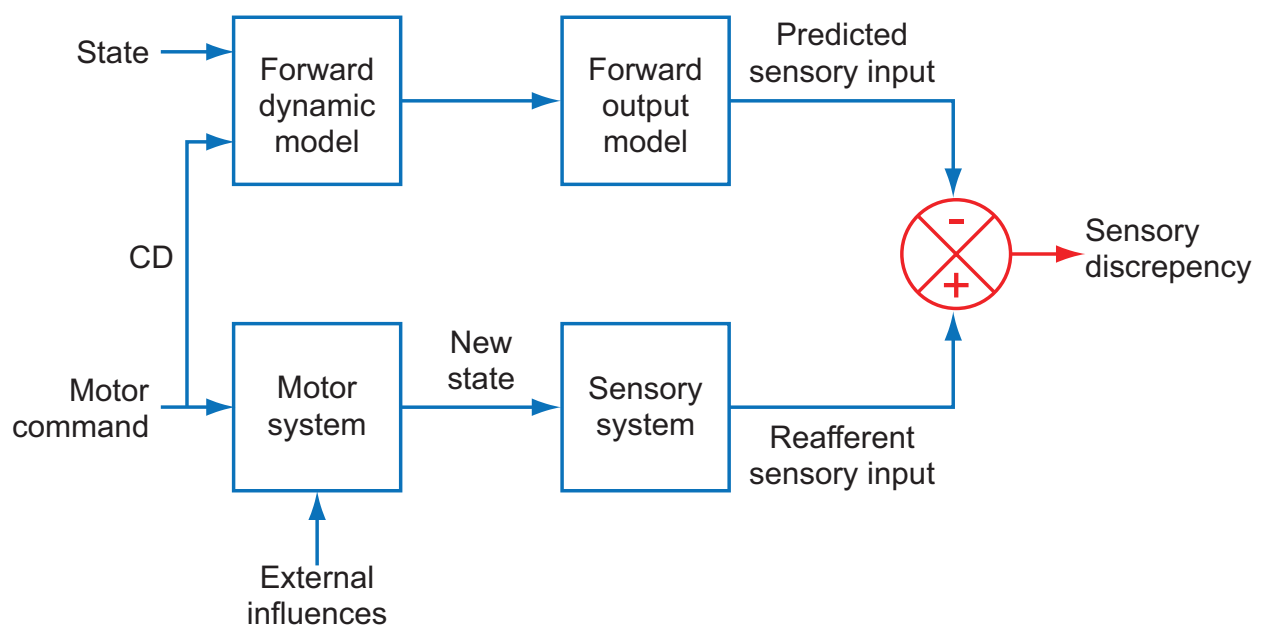

Figure 8

The forward model concept. CD (left) is combined with the current state of the system to generate a prediction of the sensory input (upper right). This prediction is compared with the actual, reafferent sensory input (lower right). Resultant discrepancies inform the brain about external influences and miscalibrations. Modified from Wolpert \& Miall (1996) with permission from Elsevier.

Afference: sensory inflow evoked by changes in the environment in contrast to afference. Cancellation mechanisms have been found in some CD circuits, for example, in electric fish (Bell 1982), but have seemed less likely in the primate visual system (Bridgeman 2007). In our experiments, the activity representing the $\mathrm{CD}$ in $\mathrm{MD}$ (a vector encoding movement) and that representing visual activity in FEF (a response field on a retinotopic map) are strikingly different. Simple cancellation makes little sense, so we find no support for this interpretation of $\mathrm{CD}$ in the primate for the visual-saccadic system.

The third and most recently promoted concept, the forward model, has been useful particularly in skeletal movement analysis (Wolpert \& Miall 1996) and has been invoked occasionally for the oculomotor system (Murthy et al. 2007, Robinson et al. 1986, Vaziri et al. 2006). Figure 8 illustrates the concept. A copy of the motor command, or CD in our terminology, is routed into a forward dynamic model. This feedback about a new movement is used to create a feedforward model of the predicted

any sensory discrepancy-similar to the cancellation interpretation of CD. A major advance of forward models over previous cancellation hypotheses, however, is the explicit recognition that the copy of the motor command, in motor coordinates, must be transformed into sensory coordinates so that it can be compared directly with the sensory input (Webb 2004, Wolpert \& Miall 1996). As we have noted above, such a motor-to-sensory transformation is exactly what we find in the FEF, which uses CD in saccadic motor coordinates to generate shifting receptive fields in visual coordinates. Hence the FEF may contain elements of a forward model (Crapse \& Sommer 2008).

Results from a variety of studies in the primate visual-saccadic system therefore support two major concepts of CD: its role in suppression mechanisms and forward models. Little evidence supports a cancellation mechanism for saccades.

\section{CD and Human Disease} forwar cons forward output model. The prediction of the sensory input is compared with the actual, reafferent sensory input to determine the extent of
For as long as CD has been recognized, researchers have speculated about the relation between CD and human disease. von Helmholtz 
(1925) described how eye muscle paralysis causes a mismatch between impaired saccades and intact "effort of will," as he called CD. He reported that the mismatch induces disturbing visual percepts, and subsequent work has refined his initial observation (Kornmueller 1931, Stevens et al. 1976). The reverse condition, in which muscles are intact but CD is lost, results from central nervous system lesions. Thalamic and parietal lesions cause CD deficits in double-step tasks, as discussed above, and parietal damage impairs the awareness of movement intentions (Sirigu et al. 2004). Frontal damage may cause CD-related deficits in perception (Teuber 1964), and visual cortex lesions can cause an illusion that the visual world is moving when the eyes move across a static background scene (Haarmeier et al. 1997).

Broader damage to CD networks, reaching beyond focal lesions, may have more devastating and long-term consequences. Schizophrenia may involve a CD disruption that could impair the self-monitoring of thoughts or actions so that they are misattributed to external sources (Feinberg 1978, Feinberg \& Guazzelli 1999). CD deficits in schizophrenia could arise from the disrupted subcorticalthalamic-cortical circuits that are known to characterize the disease (Andreasen et al. 1999). Schizophrenics are impaired in tasks requiring CD (Malenka et al. 1986), and their auditory systems show particularly strong evidence for loss of CD-related modulation (Ford et al. 2001, 2007). Neurophysiological assessment of CD circuits (e.g., the SC-MD-FEF pathway) could be performed in monkey models of schizophrenia as induced with dopaminergic or glutamatergic drugs (Condy et al. 2005, Stone et al. 2007) or fetal irradiation (Selemon et al. 2005). While a contribution of impaired $\mathrm{CD}$ to schizophrenic positive symptoms, e.g., hallucinations, is the most obvious tie-in of the function to the disease, Frith (1987) argued that impaired CD could contribute to negative and cognitive symptoms as well.

CD deficits have also been implicated in disorders with a significant motor component such as Huntington's disease (Smith et al. 2000) and developmental coordination disorder (Katschmarsky et al. 2001). We expect that further insights into the possible role of CD impairments in disease will continue to develop as circuits for $\mathrm{CD}$ in the normal brain become better understood.

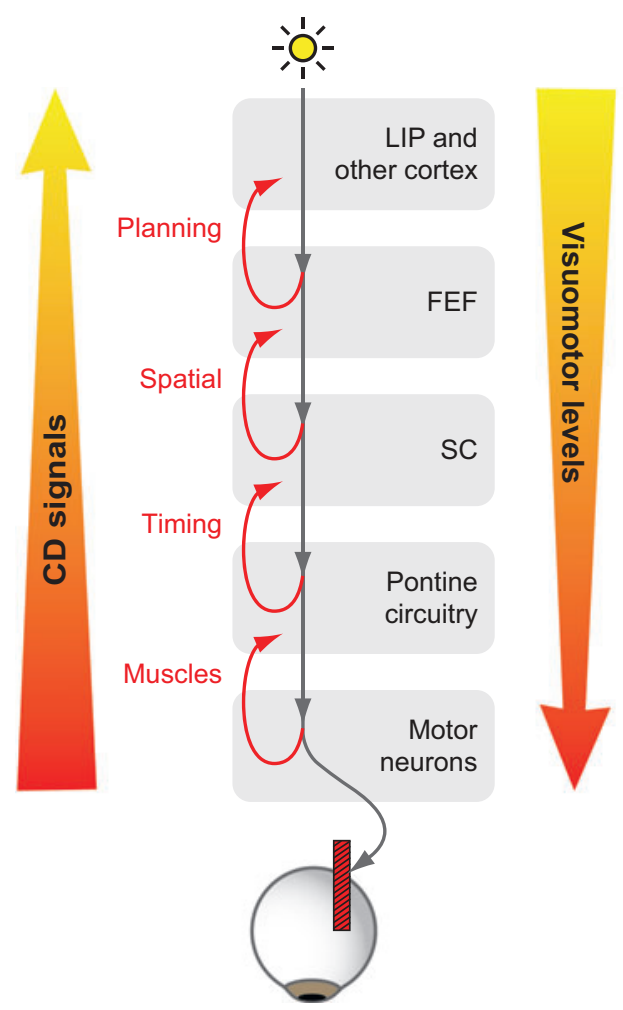

Figure 9

Hypothesized chain of saccadic CD in the primate brain. Descending visuomotor levels (right) extend from the cortical areas that detect a visual stimulus (top) to the brainstem motor neurons that innervate the muscles (bottom). Ascending CD signals may branch from any of these levels (left). The pathway that branches from SC up to the FEF conveys a CD signal rich with spatial vector information because that is the nature of the signal found in the SC and sent downstream from it. Other CD signals, branching from above and below the SC, would reflect other aspects of movement. It may be more accurate to consider $\mathrm{CD}$ as a set of signals representing the state of each saccade-related motor level, in contrast with a unitary CD signal representing the saccade itself. 


\section{CONCLUSION}

We have described the specific issue of using CD from the saccadic system to influence visual processing and have indicated more generally how CD might be involved in other brain movement systems and in their pathology. We focus on a CD pathway that emanates from the midbrain and influences the cerebral cortex.

Although we have concentrated on a CD arising from one level of a sensorimotor pathway in the primate brain, CD could arise at any of several levels along such a pathway (Figure 9). At each level, the characteristics of the descending sensorimotor signals change, and because the CD is a copy of the motor signal, the characteristics of the ascending CD would change as well. The particular visualsaccadic CD that we consider branches off at a point where saccadic vectors are represented on a retinotopic map (the SC), and this spatial CD is well suited for visuomotor functions such as shifting RFs, which require input related to that map. A CD that branches from lower in the descending pathway, in the pontine circuitry, should convey information more tightly related to saccadic timing parameters. CD copies of velocity and position signals play an important role in low-level saccadic control circuits (Robinson 1975) and may feed back to the SC (Soetedjo et al. 2002). CD signals that branch from the lowest levels would provide information about muscle innervation. At the upper extreme, CD signals may emerge from areas of cerebral cortex such as the FEF; they may convey more tentative plans to move rather than indicating distinct retinotopic vectors of the impending movement. Finally, the information at each level in the chain may diverge to influence multiple different targets. Which CD goes where would depend on the requirement of the target sensory area.

For any movement, there may be a set of CD signals; each CD conveys the state of the specific sensorimotor level from which it emerges. Although we may still speak of CD in loose terms as representing the state of a movement, it is more precise-and more beneficial to experimenters-to realize that in the primate brain, a CD signal represents the state of a movement structure such as the SC. The information content of the CD signal is limited by the signals available to the structure, and the potential targets of the CD signal are constrained by the anatomical reach of the structure's efferent pathways. Understanding these principles may be the key to identifying further CD pathways in the primate brain.

\section{DISCLOSURE STATEMENT}

The authors are not aware of any biases that might be perceived as affecting the objectivity of this review.

\section{ACKNOWLEDGEMENTS}

We thank M.E. Goldberg, C.L. Colby, R.A. Berman, T.B. Crapse, and J.P. Mayo for comments on an earlier draft of this review. The work was supported by the National Institutes of Health (EY017592) and the Alfred P. Sloan Foundation (M.A.S.), and the Intramural Research Program in the National Eye Institute, part of the National Institutes of Health (R.H.W.).

\section{LITERATURE CITED}

Andersen RA, Buneo CA. 2002. Intentional maps in posterior parietal cortex. Annu. Rev. Neurosci. 25:189-220

Andreasen NC, Nopoulos P, O'Leary DS, Miller DD, Wassink T, Flaum M. 1999. Defining the phenotype of schizophrenia: cognitive dysmetria and its neural mechanisms. Biol. Psychiatry 46:908-20 
Batista AP, Buneo CA, Snyder LH, Andersen RA. 1999. Reach plans in eye-centered coordinates. Science 285:257-60

Bell CC. 1982. Properties of a modifiable efference copy in an electric fish. F. Neurophysiol. 47:104356

Bellebaum C, Daum I, Koch B, Schwarz M, Hoffmann KP. 2005. The role of the human thalamus in processing corollary discharge. Brain 128:1139-54

Benevento LA, Fallon JH. 1975. The ascending projections of the superior colliculus in the rhesus monkey (Macaca mulatta). F. Comp. Neurol. 160:339-61

Berman RA, Heiser LM, Dunn CA, Saunders RC, Colby CL. 2007. Dynamic circuitry for updating spatial representations. III. From neurons to behavior. F. Neurophysiol. 98:105-21

Berman RA, Heiser LM, Saunders RC, Colby CL. 2005. Dynamic circuitry for updating spatial representations. I. Behavioral evidence for interhemispheric transfer in the split-brain macaque. F. Neurophysiol. 94:3228-48

Bridgeman B. 2007. Efference copy and its limitations. Comput. Biol. Med. 37:924-29

Chafee MV, Goldman-Rakic PS. 2000. Inactivation of parietal and prefrontal cortex reveals interdependence of neural activity during memory-guided saccades. F. Neurophysiol. 83:1550-66

Colby CL, Duhamel JR, Goldberg ME. 1996. Visual, presaccadic, and cognitive activation of single neurons in monkey lateral intraparietal area. 7. Neurophysiol. 76:2841-52

Colby CL, Goldberg ME. 1999. Space and attention in parietal cortex. Annu. Rev. Neurosci. 22:319-49

Condy C, Wattiez N, Rivaud-Pechoux S, Gaymard B. 2005. Ketamine-induced distractibility: an oculomotor study in monkeys. Biol. Psychiatry 57:366-72

Crapse TB, Sommer MA. 2008. The frontal eye field as a prediction map. Prog. Brain. Res. In press

Duhamel JR, Colby CL, Goldberg ME. 1992a. The updating of the representation of visual space in parietal cortex by intended eye movements. Science 255:90-92

Duhamel JR, Goldberg ME, Fitzgibbon EJ, Sirigu A, Grafman J. 1992b. Saccadic dysmetria in a patient with a right frontoparietal lesion. The importance of corollary discharge for accurate spatial behaviour. Brain 115(Pt. 5):1387-402

Feinberg I. 1978. Efference copy and corollary discharge: implications for thinking and disorders. Schizophr. Bull. 4:636-40

Feinberg I, Guazzelli M. 1999. Schizophrenia-a disorder of the corollary discharge systems that integrate the motor systems of thought with the sensory systems of consciousness. Br. $\mathcal{F}$. Psychiatry 174:196-204

Ford JM, Gray M, Faustman WO, Roach BJ, Mathalon DH. 2007. Dissecting corollary discharge dysfunction in schizophrenia. Psychophysiology 44:522-29

Ford JM, Mathalon DH, Heinks T, Kalba S, Faustman WO, Roth WT. 2001. Neurophysiological evidence of corollary discharge dysfunction in schizophrenia. Am. F. Psychiatry 158:2069-71

Frith CD. 1987. The positive and negative symptoms of schizophrenia reflect impairments in the perception and initiation of action. Psychol. Med. 17:631-48

Gaymard B, Rivaud S, Pierrot-Deseilligny C. 1994. Impairment of extraretinal eye position signals after central thalamic lesions in humans. Exp. Brain Res. 102:1-9

Goldman-Rakic PS, Porrino LJ. 1985. The primate mediodorsal (MD) nucleus and its projection to the frontal lobe. 7. Comp. Neurol. 242:535-60

Grüsser OJ. 1995. On the history of the ideas of efference copy and reafference. Clio. Med.33:35-55

Guthrie BL, Porter JD, Sparks DL. 1983. Corollary discharge provides accurate eye position information to the oculomotor system. Science 221:1193-95

Haarmeier T, Thier P, Repnow M, Petersen D. 1997. False perception of motion in a patient who cannot compensate for eye movements. Nature 389:849-52 
Hallett PE, Lightstone AD. 1976. Saccadic eye movements to flashed targets. Vis. Res. 16:107-14 Hanes DP, Schall JD. 1995. Countermanding saccades in macaque. Vis. Neurosci. 12:929-37

Heide W, Blankenburg M, Zimmermann E, Kompf D. 1995. Cortical control of double-step saccades: implications for spatial orientation. Ann. Neurol. 38:739-48

Heiser LM, Berman RA, Saunders RC, Colby CL. 2005. Dynamic circuitry for updating spatial representations. II. Physiological evidence for interhemispheric transfer in area LIP of the split-brain macaque. 7. Neurophysiol. 94:3249-58

Heiser LM, Colby CL. 2006. Spatial updating in area LIP is independent of saccade direction. 7. Neurophysiol. 95:2751-67

Katschmarsky S, Cairney S, Maruff P, Wilson PH, Currie J. 2001. The ability to execute saccades on the basis of efference copy: impairments in double-step saccade performance in children with developmental co-ordination disorder. Exp. Brain Res. 136:73-78

Kornmueller AE. 1931. Eine experimentelle anastesie der ausseren augenmusckeln am menschen und ihre auswirkungen. 7. Psychol. Neurol. Lpz. 41:354-66

Kusunoki M, Goldberg ME. 2003. The time course of perisaccadic receptive field shifts in the lateral intraparietal area of the monkey. 7. Neurophysiol. 89:1519-27

Lee PH, Sooksawate T, Yanagawa Y, Isa K, Isa T, Hall WC. 2007. Identity of a pathway for saccadic suppression. Proc. Natl. Acad. Sci. USA 104:6824-27

Lewis RF, Zee DS, Hayman MR, Tamargo RJ. 2001. Oculomotor function in the rhesus monkey after deafferentation of the extraocular muscles. Exp. Brain Res. 141:349-58

Lynch JC, Hoover JE, Strick PL. 1994. Input to the primate frontal eye field from the substantia nigra, superior colliculus, and dentate nucleus demonstrated by transneuronal transport. Exp.

Brain Res. 100:181-86

MacKay DM. 1972. Voluntary eye movements as questions. Bibl. Ophthalmol. 82:369-76

Malenka RC, Angel RW, Thiemann S, Weitz CJ, Berger PA. 1986. Central error-correcting behavior in schizophrenia and depression. Biol. Psychiatry 21:263-73

Mays LE, Sparks DL, Porter JD. 1987. Eye movements induced by pontine stimulation: interaction with visually triggered saccades. F. Neurophysiol. 58:300-17

Medendorp WP, Goltz HC, Vilis T, Crawford JD. 2003. Gaze-centered updating of visual space in human parietal cortex. F. Neurosci. 23:6209-14

Merriam EP, Genovese CR, Colby CL. 2003. Spatial updating in human parietal cortex. Neuron 39:361-73

Merriam EP, Genovese CR, Colby CL. 2007. Remapping in human visual cortex. F. Neurophysiol. 97:1738-55

Morris AP, Chambers CD, Mattingley JB. 2007. Parietal stimulation destabilizes spatial updating across saccadic eye movements. Proc. Natl. Acad. Sci. USA 104:9069-74

Murthy A, Ray S, Shorter SM, Priddy EG, Schall JD, Thompson KG. 2007. Frontal eye field contributions to rapid corrective saccades. F. Neurophysiol. 97:1457-69

Nakamura K, Colby CL. 2002. Updating of the visual representation in monkey striate and extrastriate cortex during saccades. Proc. Natl. Acad. Sci. USA 99:4026-31

Poulet JF, Hedwig B. 2007. New insights into corollary discharges mediated by identified neural pathways. Trends Neurosci. 30:14-21

Quaia C, Optican LM, Goldberg ME. 1998. The maintenance of spatial accuracy by the perisaccadic remapping of visual receptive fields. Neural Netw. 11:1229-40

Ramcharan EJ, Gnadt JW, Sherman SM. 2001. The effects of saccadic eye movements on the activity of geniculate relay neurons in the monkey. Vis. Neurosci. 18:253-58

Reppas JB, Usrey WM, Reid RC. 2002. Saccadic eye movements modulate visual responses in the lateral geniculate nucleus. Neuron 35:961-74 
Richmond BJ, Wurtz RH. 1980. Vision during saccadic eye movements. II. A corollary discharge to monkey superior colliculus. 7. Neurophysiol. 43:1156-67

Robinson DA. 1975. Oculomotor control signals. In Basic Mechanisms of Ocular Motility and Their Clinical Implications, ed. G Lennerstrand, P Bach-y-Rita, pp. 337-74. Oxford: Pergamon

Robinson DA, Gordon JL, Gordon SE. 1986. A model of the smooth pursuit eye movement system. Biol. Cybern. 55:43-57

Robinson DL, Petersen SE. 1985. Response of pulvinar neurons to real and self-induced stimulus movement. Brain Res. 338:392-94

Robinson DL, Wurtz RH. 1976. Use of an extraretinal signal by monkey superior colliculus neurons to distinguish real from self-induced stimulus movement. F. Neurophysiol. 39:852-70

Royal DW, Sary G, Schall JD, Casagrande VA. 2006. Correlates of motor planning and postsaccadic fixation in the macaque monkey lateral geniculate nucleus. Exp. Brain Res. 168:62-75

Schall JD. 2002. The neural selection and control of saccades by the frontal eye field. Philos. Trans. R. Soc. London Ser. B 357:1073-82

Schiller PH, Sandell JH. 1983. Interactions between visually and electrically elicited saccades before and after superior colliculus and frontal eye field ablations in the rhesus monkey. Exp. Brain Res. 49:381-92

Selemon LD, Wang L, Nebel MB, Csernansky JG, Goldman-Rakic PS, Rakic P. 2005. Direct and indirect effects of fetal irradiation on cortical gray and white matter volume in the macaque. Biol. Psychiatry 57:83-90

Sirigu A, Daprati E, Ciancia S, Giraux P, Nighoghossian N, et al. 2004. Altered awareness of voluntary action after damage to the parietal cortex. Nat. Neurosci. 7:80-84

Smith MA, Brandt J, Shadmehr R. 2000. Motor disorder in Huntington's disease begins as a dysfunction in error feedback control. Nature 403:544-49

Soetedjo R, Kaneko CR, Fuchs AF. 2002. Evidence that the superior colliculus participates in the feedback control of saccadic eye movements. F. Neurophysiol. 87:679-95

Sommer MA, Wurtz RH. 1998. Frontal eye field neurons orthodromically activated from the superior colliculus. 7. Neurophysiol. 80:3331-35

Sommer MA, Wurtz RH. 2002. A pathway in primate brain for internal monitoring of movements. Science 296:1480-82

Sommer MA, Wurtz RH. 2004a. What the brain stem tells the frontal cortex. I. Oculomotor signals sent from superior colliculus to frontal eye field via mediodorsal thalamus. F. Neurophysiol. 91:1381-402

Sommer MA, Wurtz RH. 2004b. What the brain stem tells the frontal cortex. II. Role of the SC-MD-FEF pathway in corollary discharge. F. Neurophysiol. 91:1403-23

Sommer MA, Wurtz RH. 2006. Influence of the thalamus on spatial visual processing in frontal cortex. Nature 444:374-77

Sparks DL, Hartwich-Young R. 1989. The neurobiology of saccadic eye movements. The deep layers of the superior colliculus. Rev. Oculomot. Res. 3:213-56

Sparks DL, Mays LE. 1983. Spatial localization of saccade targets. I. Compensation for stimulation-induced perturbations in eye position. 7. Neurophysiol. 49:45-63

Sperry RW. 1950. Neural basis of the spontaneous optokinetic response produced by visual inversion. 7. Comp. Physiol. Psychol. 43:482-89

Steinbach MJ. 1987. Proprioceptive knowledge of eye position. Vis. Res. 27:1737-44

Stevens JK, Emerson RC, Gerstein GL, Kallos T, Neufeld GR, et al. 1976. Paralysis of the awake human: visual perceptions. Vis. Res. 16:93-98

Stone JM, Morrison PD, Pilowsky LS. 2007. Glutamate and dopamine dysregulation in schizophrenia-a synthesis and selective review. F. Psychopharmacol. 21:440-52 
Teuber HL. 1964. The riddle of the frontal lobe function in man. In The Frontal Cortex and Behavior. pp. 410-444. New York: McGraw-Hill

Thiele A, Henning P, Kubischik M, Hoffmann KP. 2002. Neural mechanisms of saccadic suppression. Science 295:2460-62

Tolias AS, Moore T, Smirnakis SM, Tehovnik EJ, Siapas AG, Schiller PH. 2001. Eye movements modulate visual receptive fields of V4 neurons. Neuron 29:757-67

Umeno MM, Goldberg ME. 1997. Spatial processing in the monkey frontal eye field. I. Predictive visual responses. 7. Neurophysiol. 78:1373-83

Umeno MM, Goldberg ME. 2001. Spatial processing in the monkey frontal eye field. II. Memory responses. F. Neurophysiol. 86:2344-52

Vaziri S, Diedrichsen J, Shadmehr R. 2006. Why does the brain predict sensory consequences of oculomotor commands? Optimal integration of the predicted and the actual sensory feedback. 7. Neurosci. 26:4188-97

von Helmholtz H. 1925 [1910]. Helmboltz's Treatise on Physiological Optics. Transl. JPC Southall. New York: Opt. Soc. Am. 3rd ed.

von Holst E, Mittelstaedt H. 1950. Das reafferenzprinzip. Wechselwirkungen zwischen centralnervensystem und peripherie. Naturwissenschaften 37:464-76

Walker MF, FitzGibbon EJ, Goldberg ME. 1994. Predictive visual responses in monkey superior colliculus. In Contemporary Ocular Motor and Vestibular Research: A Tribute to David A. Robinson, ed. U Büttner, T Brandt, A Fuchs, D Zee, pp. 512-19. Stuttgart, Germany: Thieme

Wang X, Zhang M, Cohen IS, Goldberg ME. 2007. The proprioceptive representation of eye position in monkey primary somatosensory cortex. Nat. Neurosci. 10:640-46

Webb B. 2004. Neural mechanisms for prediction: Do insects have forward models? Trends Neurosci. 27:278-82

Wolpert DM, Miall RC. 1996. Forward models for physiological motor control. Neural Netw. 9:1265-79

Wurtz RH, Sommer MA. 2004. Identifying corollary discharges for movement in the primate brain. Prog. Brain Res. 144:47-60

\section{RELATED RESOURCES}

Bell, CC. 2008. Cerebellum-like structures. Annu. Rev. Neurosci. 31:1-25

Bridgeman B. 2007. Efference copy and its limitations. Comput. Biol. Med. 37:924-29

Colby CL, Goldberg ME. 1999. Space and attention in parietal cortex. Annu. Rev. Neurosci. 22:319-49

Poulet JF, Hedwig B. 2007. New insights into corollary discharges mediated by identified neural pathways. Trends Neurosci. 30:14-21 
西

Annual Review of

Neuroscience

Contents

Volume 31, 2008

Cerebellum-Like Structures and Their Implications for Cerebellar Function

Curtis C. Bell, Victor Han, and Nathaniel B. Sawtell ................................ 1

Spike Timing-Dependent Plasticity: A Hebbian Learning Rule

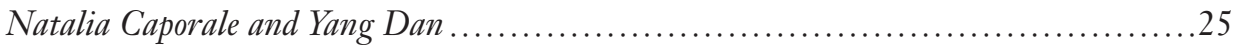

Balancing Structure and Function at Hippocampal Dendritic Spines

Fennifer N. Bourne and Kristen M. Harris....

Place Cells, Grid Cells, and the Brain's Spatial Representation System

Edvard I. Moser, Emilio Kropff, and May-Britt Moser

Mitochondrial Disorders in the Nervous System

Salvatore DiMauro and Eric A. Schon

Vestibular System: The Many Facets of a Multimodal Sense

Dora E. Angelaki and Katbleen E. Cullen

Role of Axonal Transport in Neurodegenerative Diseases

Kurt 7. De Vos, Andrew 7. Grierson, Steven Ackerley, and Christopher C.7. Miller ... 151

Active and Passive Immunotherapy for Neurodegenerative Disorders

David L. Brody and David M. Holtzman

Descending Pathways in Motor Control

Roger N. Lemon

Task Set and Prefrontal Cortex

Katsuyuki Sakai

Multiple Sclerosis: An Immune or Neurodegenerative Disorder?

Bruce D. Trapp and Klaus-Armin Nave

Multifunctional Pattern-Generating Circuits

K.L. Briggman and W.B. Kristan, Fr.

Retinal Axon Growth at the Optic Chiasm: To Cross or Not to Cross

Timothy 7. Petros, Alexandra Rebsam, and Carol A. Mason 
Brain Circuits for the Internal Monitoring of Movements

Marc A. Sommer and Robert H. Wurtz

Wnt Signaling in Neural Circuit Assembly

Patricia C. Salinas and Yimin Zou

Habits, Rituals, and the Evaluative Brain

Ann M. Graybiel

Mechanisms of Self-Motion Perception

Kenneth H. Britten

Mechanisms of Face Perception

Doris Y. Tsao and Margaret S. Livingstone

The Prion's Elusive Reason for Being Adriano Aguzzi, Frank Baumann, and Fuliane Bremer ....

Mechanisms Underlying Development of Visual Maps and Receptive Fields

Andrew D. Huberman, Marla B. Feller, and Barbara Chapman

Neural Substrates of Language Acquisition

Patricia K. Kubl and Maritza Rivera-Gaxiola

Axon-Glial Signaling and the Glial Support of Axon Function

Klaus-Armin Nave and Bruce D. Trapp

Signaling Mechanisms Linking Neuronal Activity to Gene Expression and Plasticity of the Nervous System Steven W. Flavell and Michael E. Greenberg 563

\section{Indexes}

Cumulative Index of Contributing Authors, Volumes 22-31

Cumulative Index of Chapter Titles, Volumes 22-31 .... 595

\section{Errata}

An online log of corrections to Annual Review of Neuroscience articles may be found at http://neuro.annualreviews.org/ 